\title{
Applying Virtual Reality to Improve the Construction Logistics of High-rise Modular Integrated Construction
}

\author{
Enoch H.L. Cheung ${ }^{1}$ And S. Thomas $\mathrm{Ng}^{2 *}$ \\ ${ }^{1}$ Student, Department of Civil Engineering, The University of Hong Kong \\ ${ }^{2}$ Associate Dean, Faculty of Engineering, The University of Hong Kong \\ *Corresponding author's e-mail: tstng@hku.hk
}

\begin{abstract}
Modular and offsite construction is becoming increasingly popular around the world. In Hong Kong, a modular integration construction (MiC) method is identified as a pragmatic approach to speed up the housing construction program and to solve the productivity and manpower problems of the industry. Using the MiC, virtually all the construction works including the finishing as well as the mechanical and electrical installation are completed offsite. The MiC units are then delivered to and installed on site. While the MiC can shift the risks of construction projects to the factories, this construction method is not without challenges. This is particularly the case for Hong Kong as most of the construction sites in the city are cramped due to the high-density urban environment. The problem is aggravated when every modular unit is unique and they are time consuming to produce. Any damages to the $\mathrm{MiC}$ components during the lifting process could seriously affect the entire construction sequence under a just-in-time management philosophy. Therefore, it is imperative to plan and monitor the logistics carefully when the $\mathrm{MiC}$ technique is used. To reduce any human errors and increase the efficiency and accuracy of the lifting process, a virtual reality (VR) approach may be adopted to simulate the construction logistics of MiC construction and train the crane operators. In this paper, a VR model is developed to simulate the construction of a highrise residential building in a confined site. Various functions are built into the VR model to support the decisions pertinent to lifting logistics planning. In this paper, the design considerations and functions of the VR model are identified through a series of interviews. Moreover, the validation interviews help unveil the potentials and pitfalls of the developed VR model.
\end{abstract}

\section{KEYWORDS}

Modular integrated construction; Construction logistics; Virtual reality; Lifting process

\section{INTRODUCTION}

Growing population and economic growth have led to a strong demand for construction facilities worldwide with no exception to Hong Kong. In order to expedite the construction program, many projects in particular those residential buildings are relying heavily on the modular and off-site technologies. Taking the public rental housing blocks in Hong Kong as an example, prefabricated façade, staircase, bathroom and kitchen have been uses for decades.

To further improve construction productivity and safety, a modular integrated construction (MiC) technique has received significant attention these few years. MiC or prefabricated prefinished 
volumetric construction (PPVC) have been used for constructing high-rise residential apartments, hotels, student dormitories, etc. in the United Kingdom, Singapore and Hong Kong. According to Lim (2016), a hotel project using the MiC or PPVC technique has resulted in a 50\% and 33\% drop in manpower and time when compared to convention method.

Under the MiC concept, a building is divided into standalone offsite prefabrication components of optimal transportable and liftable sizes (Hwang et al., 2018a). These MiC components can be made of various materials including but not limited to steel and concrete so as to maintain their structural integrity during transportation and lifting as well as after the installation. To maximize the benefits of factory production, the MiC components are prefinished with relevant finishings and fixtures before delivery (Hwang et al., 2018b). The only tasks on site would be inspection and patching up any defective parts before those $\mathrm{MiC}$ components are fixed together and the services are connected (Olearczyk et al., 2014).

Despite its advantages (Cranes Today, 2016), adopting the MiC technique is not without challenges. This is especially the case in a high-density urban environment setting, as there is an absence of space on site for storing any buffer stocks of MiC components. Besides, the logistics of the MiC components between the factories (which are usually located in mainland China) to the construction site or even the onsite logistics would also require lots of serious considerations as those $\mathrm{MiC}$ components are massive to maneuver and handle. Any misjudgment in planning would not only lead to project delay, but it could also pose substantial safety risks to workers and nearby properties and parties.

Recent advance in virtual reality (VR) may open up new ways to help plan and model the MiC projects. Through VR, an immersive environment is constructed to users to interact with the objects in the virtual environment in order to simulate various possible scenarios (Schechter, 2015). VR provides a platform for users to interact with. This would help minimize the unnecessary and costly human errors when it comes to the real construction work.

In this paper, the potential of using VR to improve the MiC planning and construction is explored. Of various issues, the lifting of MiC components appears to be one of the most important stages in the construction of this type of facility. Therefore, a VR model related to the planning and usage of tower crane is developed in this paper. The following sections will describe the development of the VR model, the functionality of the VR model, and the preliminary results.

\section{RESEARCH METHODOLOGY}

The research was conducted into two major stages. In the first stage, literature was reviewed conducted to gain in-depth knowledge related to $\mathrm{MiC}$ and tower crane operation. The literature provided the details of tower crane planning and operation, while the code of practices highlighted the precautions and rules governing tower crane operation. Interviews were then carried out to uncover the challenges associated with planning and lifting $\mathrm{MiC}$ components. In the second stage, the VR model was developed using a commonly used VR tool - Unity3D ${ }^{\mathrm{TM}}$.

To develop an immersive environment which closely reassembles the reality, a real MiC project was selected as the case project. The project is a 28-story residential building in Australia. 
Drawings related to the building as well as the details of construction sequence had been solicited from internet, and they were in turn used for building the VR model. Besides, the challenges identified from literature and through interviews in particular that related to the logistic planning were then integrated to the VR model to create the what-if scenarios.

The VR model was built using Unity $3 \mathrm{D}^{\mathrm{TM}}$ and the programming language $\mathrm{C} \#$. When building the VR scenario, various game objects downloaded from the Unity Asset Store were used to reduce the development time. These game objects including the material, elements, machines, etc. were then imported to Unity3 $\mathrm{D}^{\mathrm{TM}}$ for integration with the virtual environment. The scripts for defining user interaction were then programed using $\mathrm{C \#}$, and these scripts were linked to the game objects to provide the necessary commands. Finally, the VR model was exported for usage.

After the model was completed, it was tested and evaluated in both the windows and Mac operating systems. The evaluation was conducted based on the users' feedback on the VR model. In this regard, the second round of interview was conducted to gain feedback from professionals in the construction industry. Through which, the feasibility of implementing the proposed VR model in the construction industry was evaluated and the limitations of the model were also addressed.

\section{UNITY3DTM}

Unity $3 \mathrm{D}^{\mathrm{TM}}$ is composed of 3 major components, including game objects, scripts and animations. Game objects are the basic elements in Unity3 $\mathrm{D}^{\mathrm{TM}}$ that builds up the virtual environment. The game objects are arranged under the hierarchy window on the left hand side as shown in Figure 1. This allows the programmer to manage the game objects in an organized manner. For instance, "towerCrane" shown in the hierarchy window refers to the tower crane in the scene, with the cabin and base under its hierarchy (Figure 1).

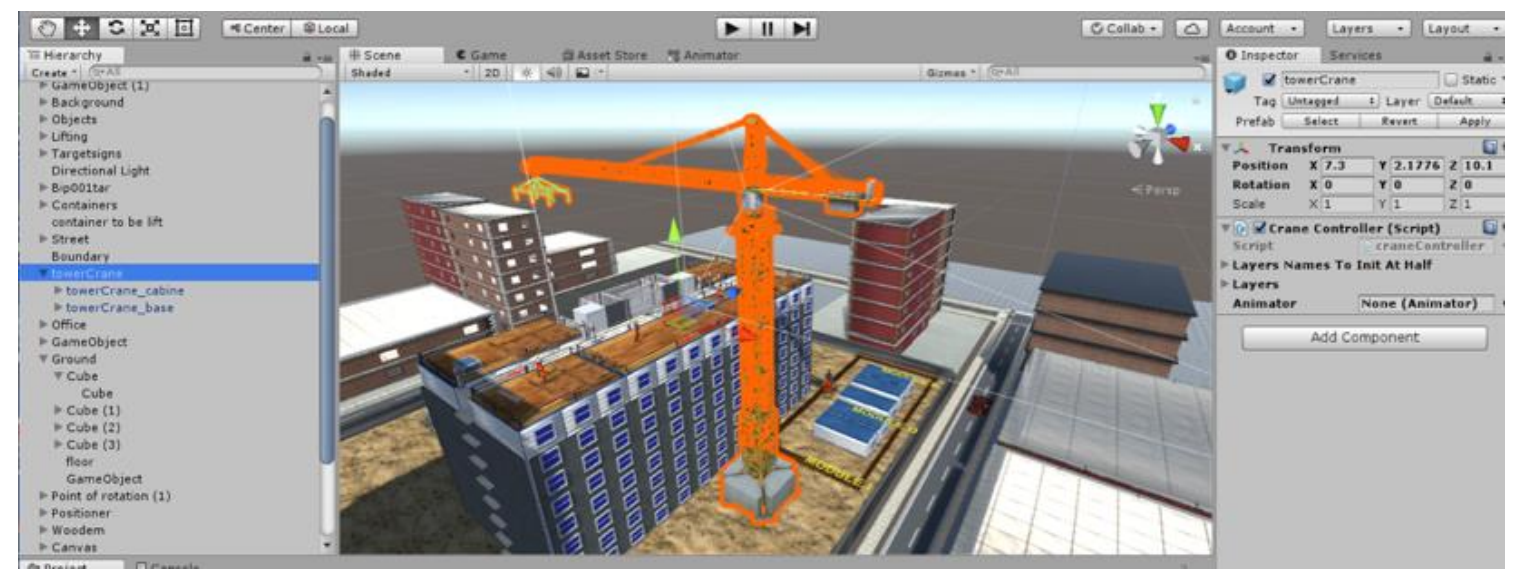

Figure 1. Tower crane in hierarchy window

As it is difficult to model every single game object from the start, a wide variety of game objects like buildings, tower cranes and roads can be downloaded from the Unity Asset Store. For example, the buildings surrounding the construction site were imported from the "City Tools Pack" in the Unity Asset Store. It contains the basic components of a building, viz. floor, walls and ceiling, which were used to construct a building model as shown in Figure 2. 


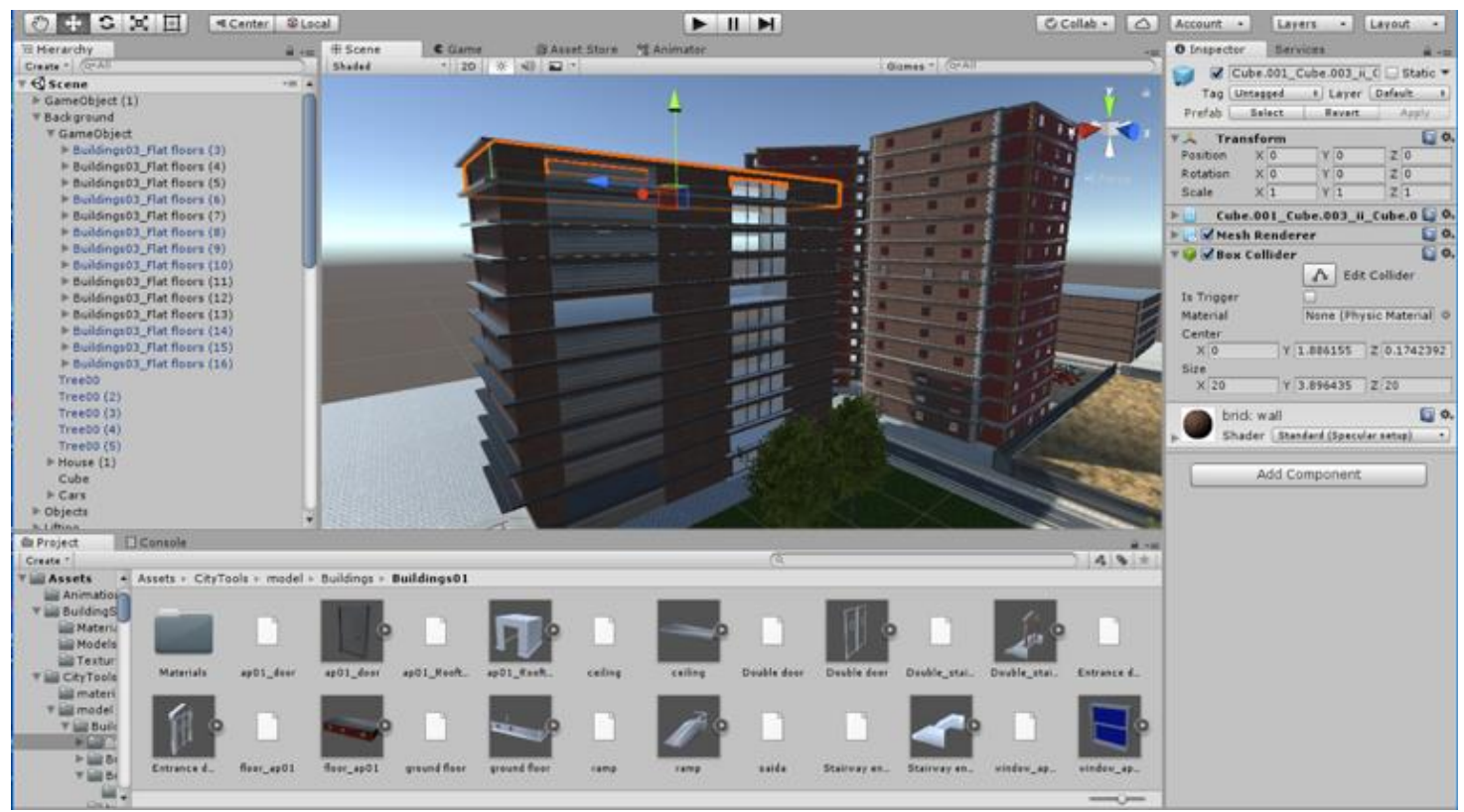

Figure 2. Building constructed from the "City Tool Pack" in the Asset Store

In order to make the VR model more realistic, interactions have to be assigned to the game objects. "Script" is for responding the input from the users and displaying the events in the gameplay to occur in the next step (Figure 3). Unity supports C\# and Java script, and C\# was chosen as the coding language in this project. Through assigning the scripts to the game objects, the player can interact with the virtual environment. For instance, the player can control the tower crane by pushing the direction buttons.

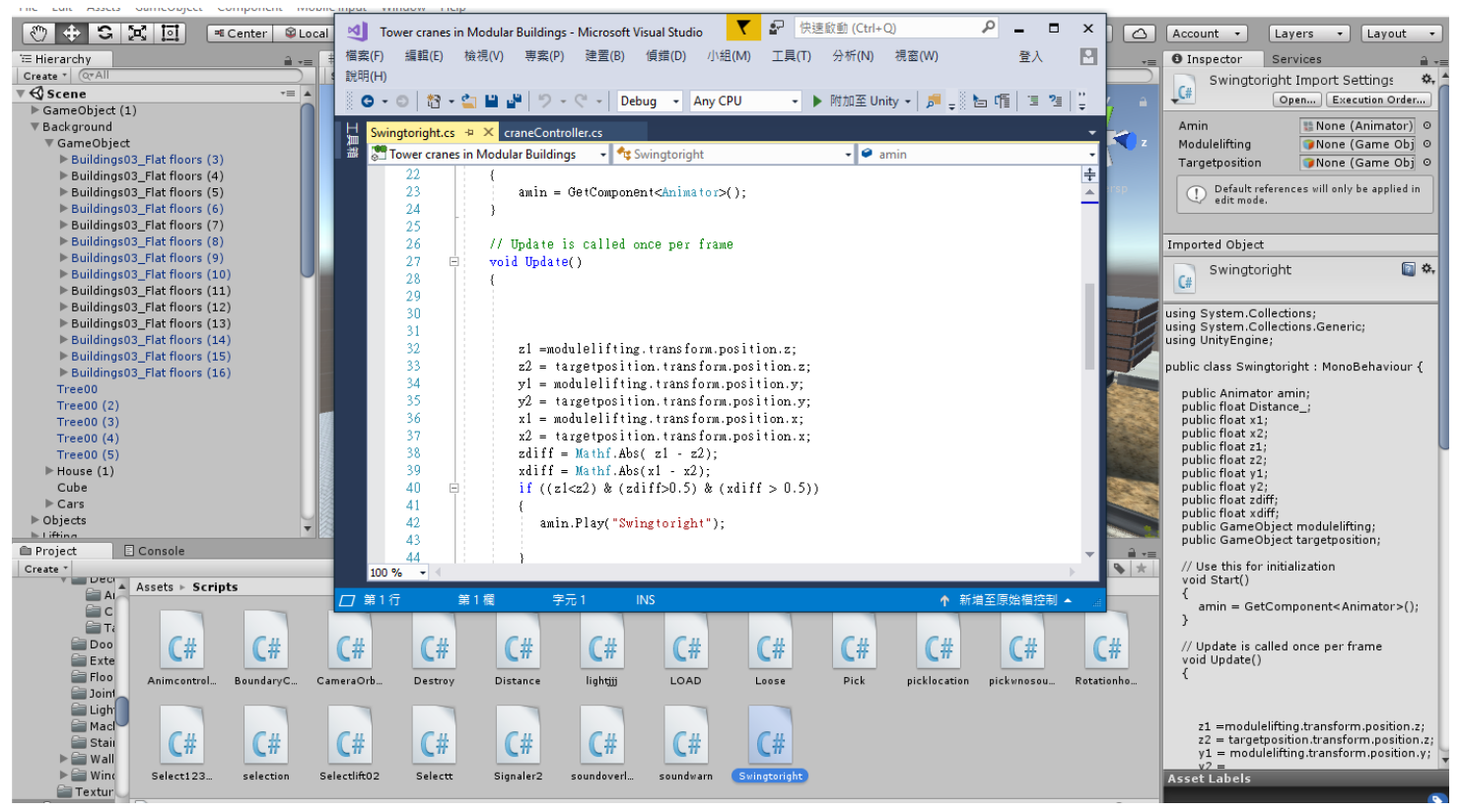

Figure 3. C\# scripts in Unity $3 \mathrm{D}^{\mathrm{TM}}$

In this project, a total of 10 scripts are used, 8 scripts are self-written to simulate the operation of tower cranes, movement of signaler and logistics of modular construction (Figure 3), the remaining 
2 scripts for the camera and part of tower crane movement were downloaded from the Unity website. The scripts help improve the interactivity and quality of the VR model.

Animations are used in Unity3 $\mathrm{D}^{\mathrm{TM}}$ to define the movements of the game objects. The animations of game objects are based on the orders defined in the animator clip. As shown in Figure 4, the animator clip can be used to define the rotation, position and scale of game objects. The relative coordinate, angle and scale can be manually set to produce the animation effect. For example, the crane jib rotational movement was defined to rotate about the $\mathrm{z}$-axis at the angular velocity of $360 \%$ minute in order to simulate the rotation of the tower crane jib.

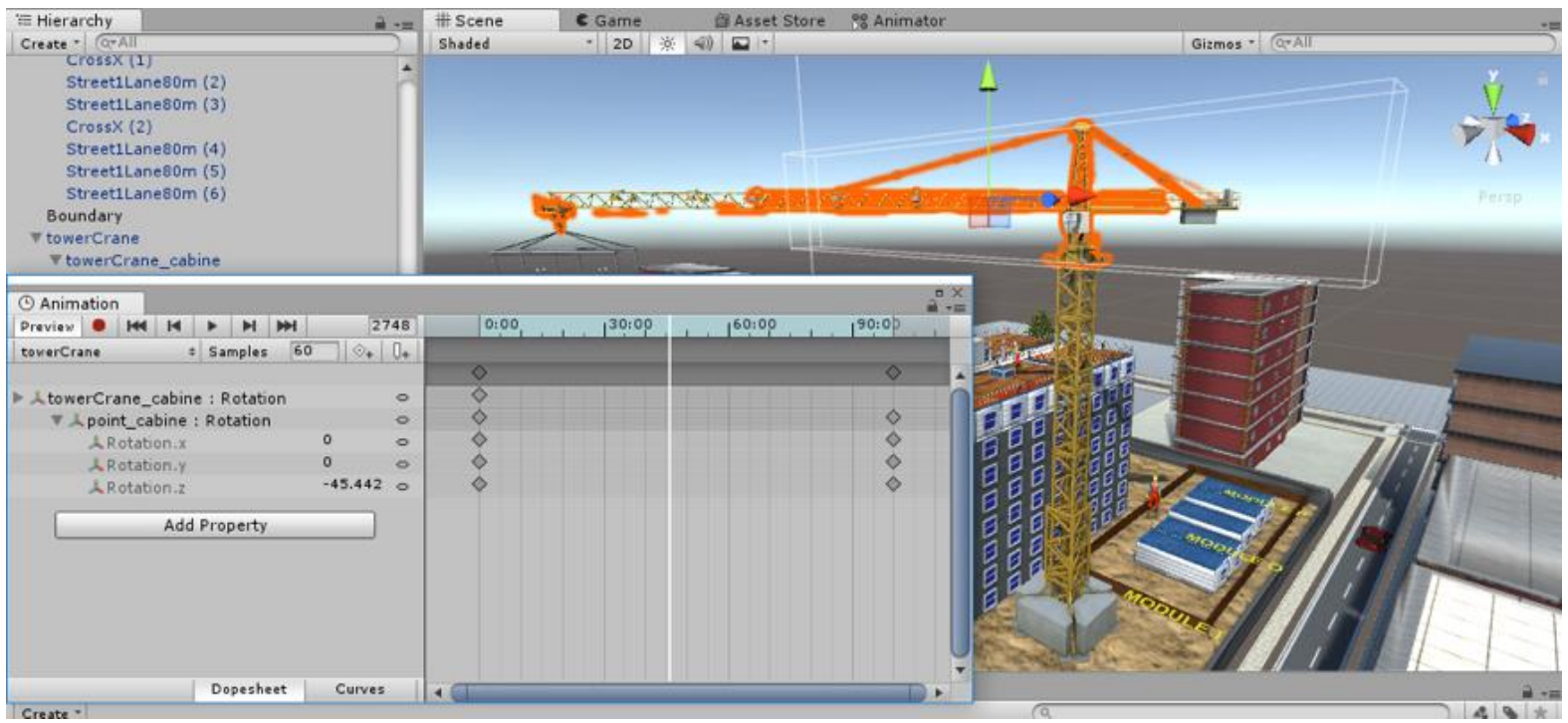

Figure 4. Animator clip for rotation movement of tower crane

For any Animator Clip, it must be set reference to an Animator Controller. This Animator controller contains references to one or more Animation Clips. By detecting the input from the user, the Animator Controller can direct towards the required Animation Clip. For instance, if the user pushes the "left" button on the keyboard, the tower crane jib would rotate anticlockwise accordingly. In this project, 9 Animator Clips and 3 Animator Controllers are used, with 6 Animator Clips are produced originally to simulate the movement of tower crane and signaler. The remaining 3 Animator Clips are downloaded from the Unity Asset Store.

\section{VIRTUAL REALITY MODEL DEVELOPED}

In order to make the VR model more realistic, the virtual environment was built based on the real construction site and surroundings environment. Figure 5 shows the case building and the surrounding environment obtained from Google Earth ${ }^{\mathrm{TM}}$, while Figure 6 displays the virtual environment constructed using Unity3 $\mathrm{D}^{\mathrm{TM}}$.

For the surrounding environment, there are two buildings, an open area and a car park next to the case building. Hence it is important to ensure that the lifting operations are within the site boundary to prevent causing any damages to the surrounding properties. There are also two doublecarriageway roads abutting the site of the case building, which provide access to the site. 


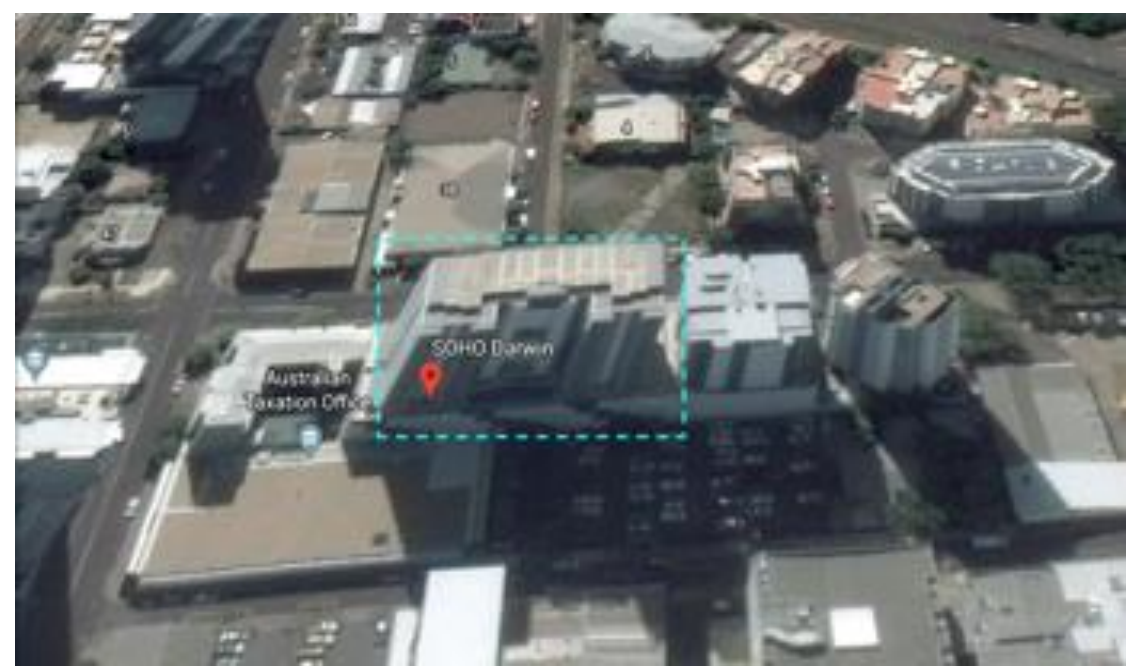

Figure 5. Existing SOHO tower from Google Earth ${ }^{\mathrm{TM}}$

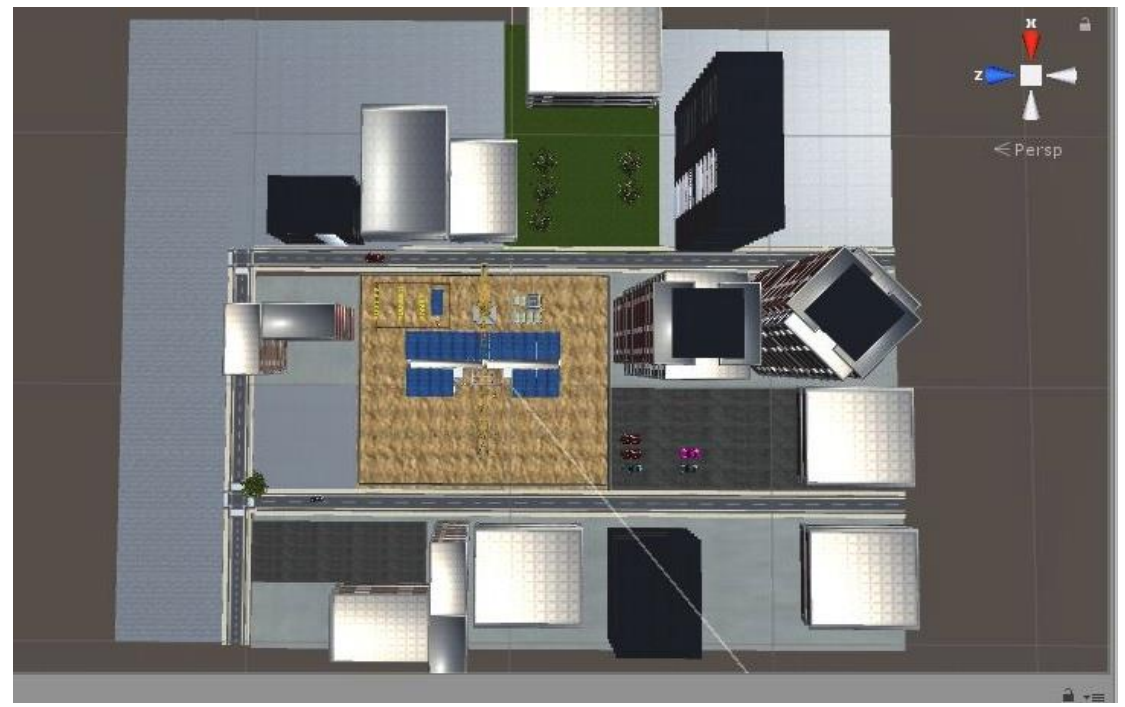

Figure 6. Virtual construction site constructed in Unity3 $\mathrm{D}^{\mathrm{TM}}$

\section{LOGISTIC PLANNING}

One of the functions of the developed VR model is to analyze the logistics of module lifting to different locations. Given the large size and heavy weight of modules, it would be risky for the module to have insufficient clearance between the modules and obstacles, and this may not have been taken into account in the BIM model or other assessment. By involving the subcontractors, crane operator and main contractor in site planning at an earlier stage, this VR model provides a platform to evaluate whether the lifting path is feasible under different stages of the project.

In the developed VR model, it allows users to control the tower crane manually to lift the MiC components from different starting points to different destinations. Through which, it brings the vision of the tower crane operation to life. To make the VR model more realistic, there are two special features, namely the target position selection and the signaler guidance. 
Firstly, the user is allowed to select different target positions to place the module. For simplicity, only 5 targets are available for selection by pressing " 1 " to " 5 " on the keyboard, which are "TARGET 1", "TARGET 2", "TARGET 3", "TARGET 4" and "TARGET 5". The site is divided into 3 working areas highlighted in red, blue and green as shown in Figure 7. Once a specific target position is selected, the other areas will be transformed to "work in progress". The script "Select_Target" can detect users' input and change the corresponding position of the "work in progress" areas, the signaler and the light pointer. This is to simulate the day cycle construction method in the construction of MiC projects.

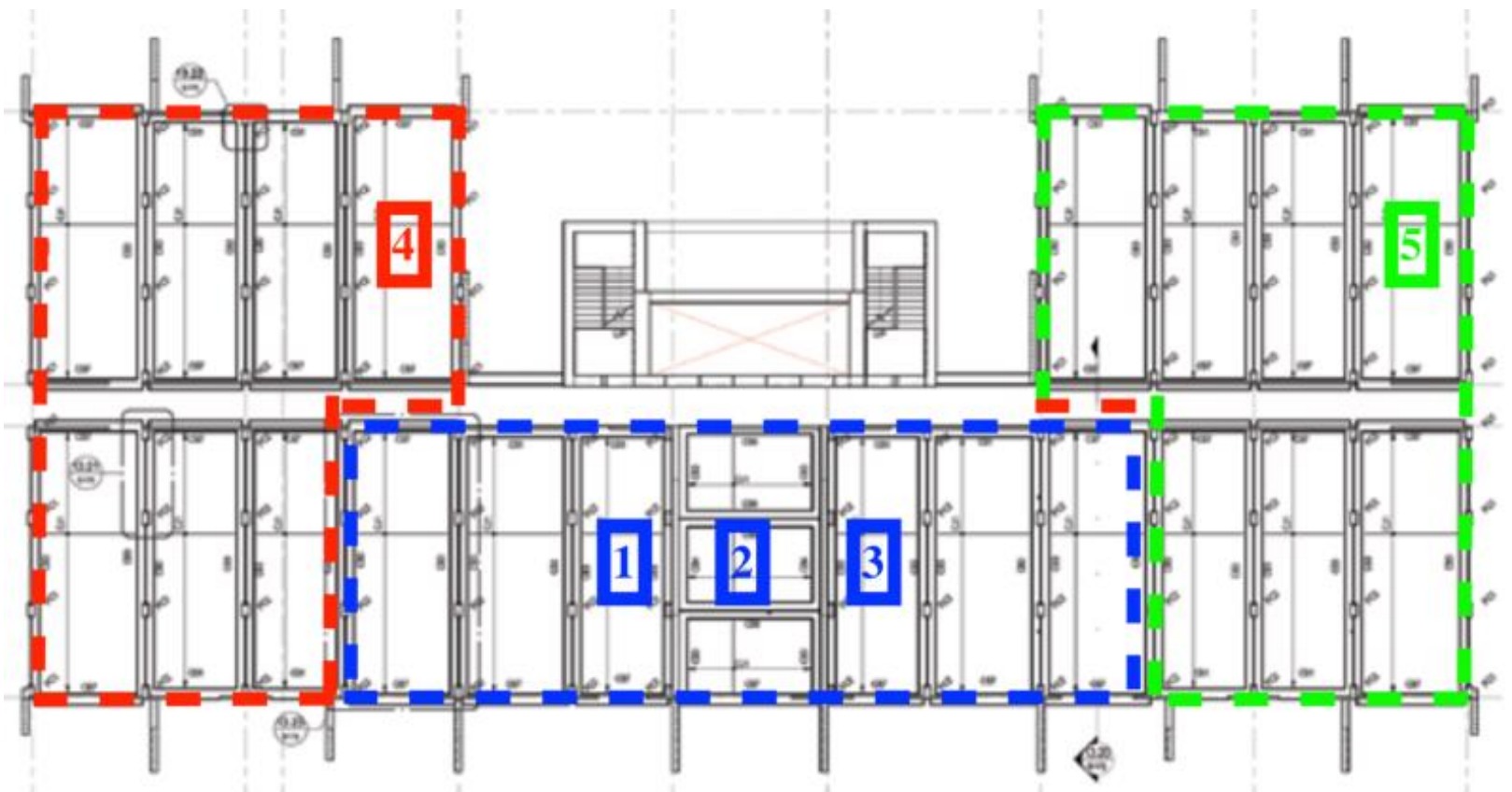

Figure 7. The 3 work areas and 5 target positions

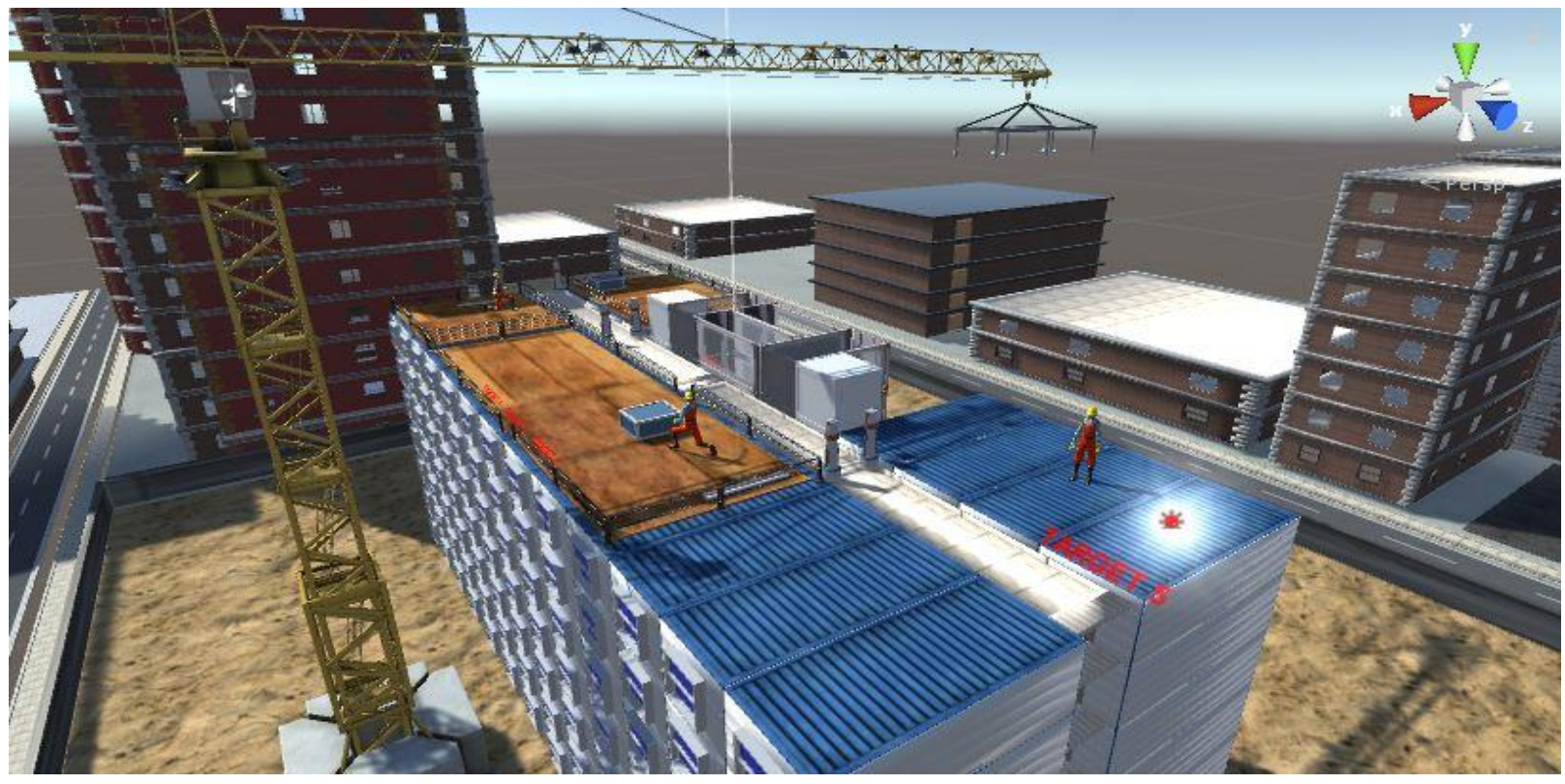

Figure 8. The 3 working areas and 5 target positions in the VR model 
If the user wants to lift the module to the position of "TARGET 5", he/she shall press " 5 " on the keyboard. Then, the light pointer will move to "TARGET 5" and the signaler will stand beside the module, with the other areas at "TARGET 1-3" and "TARGET 4" transformed into the "work in progress" areas covered with timber formwork (Figure 8). As there are some construction workers and equipment in the "work in progress" areas, they will fall off the building if the MiC component being lifted collides with the workers and or equipment during lifting.

\section{CONCLUSIONS}

The use of modular integrated construction (MiC) will increasingly popular in Hong Kong as this construction technique can help resolve the labor shortage problem, increase construction productivity, and improve site safety. However, extensive lifting of MiC components could pose a risks to this type of project as improper logistic planning and lifting could lead to fatal accidents.

In the paper, a virtual reality (VR) model has been developed to help improve the logistic planning. Various scenarios have been built into the VR model to allow planners and crane drivers to simulate the consequences of improper logistic planning and lifting. Apart from logistic planning, the VR model developed also provide simulation for four other functions, namely: tower crane planning; precautions in tower crane lifting; safety measures of tower crane operation; and automatic safe load indicator.

While the VR model presented in this paper is far from perfect, improvements are needed to include more realistic functions and problem scenarios. Given time to refine the VR model, it can serve as a planning alert and/or training tools for contractor, planner, safety personnel and crane operators to make them fully acquainted of this rather new construction technique.

\section{REFERENCES}

Cranes Today (2016). "Building blocks." Cranes Today Magazine, 11 November 2016 <http://www.cranestodaymagazine.com/features/building-blocks-5660673/> (Jan. 29, 2017).

Schechter, S. (2015). "What is virtual reality?" Marxent Labs, 3 June 2015, $<$ https://www.marxentlabs.com/what-is-virtual-reality/> (Jan. 29, 2017).

Lim, S. (2016). "Lego-like building method to speed up Crowne Plaza Changi Airport hotel extension." Today, 11 February 2016, <https://www.todayonline.com/singapore/extensioncrowne-plaza-changi-airport-slated-completion-june> (Jan. 29, 2017).

Hwang, B.G., Shan, M., and Looi, K.Y. (2018a). "Key constraints and mitigation strategies for prefabricated prefinished volumetric construction." Journal of Cleaner Production, 183, 183-193.

Hwang, B.G., Shan, M., and Looi, K.Y. (2018b). "Knowledge-based decision support system for prefabricated prefinished volumetric construction." Automation in Construction, 94, 168178.

Olearczyk, J., Al-Hussein, M., and Bouferguunne, A. (2014). "Evolution of the crane selection and on-site utilization process for modular construction multilifts." Automation in Construction, 43, 59-72. 